\title{
Nontuberculous Mycobacterial Lung Disease Accompanied by Organizing Pneumonia
}

\author{
Yasuharu Nakahara ${ }^{1}$, Yasutaka Oonishi ${ }^{1}$, Junji Takiguchi ${ }^{2}$, Akie Morimoto ${ }^{1}$, \\ Katsunari Matsuoka ${ }^{3}$, Naoko Imanishi $^{3}$, Takanori Higashino ${ }^{4}$, Rokuro Mimura ${ }^{5}$, \\ Tetsuji Kawamura ${ }^{1}$ and Yoshiro Mochiduki ${ }^{1}$
}

\begin{abstract}
We herein report our experience with patients who had nontuberculous mycobacterial lung disease (NTM disease) accompanied by organizing pneumonia (OP). Out of 98 NTM disease patients who had undergone a biopsy or surgical resection, 11 patients had OP that was revealed histologically. After excluding six patients who had OP-related diseases (idiopathic interstitial pneumonia, rheumatoid arthritis, etc.), the remaining five patients were studied. Two of them (a 73-year-old man and a 66-year-old woman) showed common clinical feature: acute-onset symptoms of cough and fever, infiltrating shadows and dramatic improvement following treatment with a corticosteroid and anti-mycobacterial therapy. Our cases demonstrate that NTM disease is sometimes accompanied by OP histologically, and some such cases show common clinical features.
\end{abstract}

Key words: nontuberculous mycobacteria, organizing pneumonia, histology, acute, infiltrating shadow, steroid

(Intern Med 54: 945-951, 2015)

(DOI: 10.2169/internalmedicine.54.3616)

\section{Introduction}

The clinical spectrum of nontuberculous mycobacterial lung disease (NTM disease) in immunocompetent patients includes fibrocavitary disease, nodular/bronchiectatic disease, solitary pulmonary nodules and hot tub lung (1). Recently, cases with certain clinical features have been reported: NTM disease accompanied by histologically-proven organizing pneumonia (OP), also known as bronchiolitis obliterans organizing pneumonia $(2,3)$, in which patients developed acute or subacute symptoms with infiltrates on chest images, and improved dramatically following treatment with a combination of a corticosteroid and antimycobacterial therapy (4-6). We also found similar cases among our NTM disease patients. No description about these clinical features is currently found in the official
American Thoracic Society (ATS)/Infectious Diseases Society of America (IDSA) statement regarding the diagnosis, treatment and prevention of nontuberculous mycobacterial diseases (1).

Marchevsky et al. (7) reported in their study of Mycobacteria-positive open-lung biopsy specimens from 40 patients that the specimens of three patients with bilateral diffuse infiltrating shadows and no underlying conditions exhibited OP histologically. We thought that their disease entity might be the same as that of the cases mentioned above. On the other hand, they reported that histological OP was also seen in a patient with multiple discrete infiltrates (7), which suggests that NTM disease accompanied by histological OP may show various radiological patterns other than diffuse infiltrations. To clarify the clinical and radiological features of patients with coexisting NTM disease and histological OP, we reviewed our NTM disease patients in whom

${ }^{1}$ Department of Respiratory Medicine, National Hospital Organization Himeji Medical Center, Japan, ${ }^{2}$ Department of Respiratory Medicine, Kobe Laborers' Hospital, Japan, ${ }^{3}$ Department of Respiratory Surgery, National Hospital Organization Himeji Medical Center, Japan, ${ }^{4}$ Department of Radiology, National Hospital Organization Himeji Medical Center, Japan and ${ }^{5}$ Department of Pathology, National Hospital Organization Himeji Medical Center, Japan

Received for publication July 7, 2014; Accepted for publication August 19, 2014

Correspondence to Dr. Yasuharu Nakahara, nakahary@hmj-net.hosp.go.jp 
Table. Laboratory Findings on the Time Point of Histological Examination

\begin{tabular}{|c|c|c|c|c|c|c|c|c|c|c|c|c|c|}
\hline & $\underset{\times 10^{4} / \mu \mathrm{L}}{\mathrm{RBC}}$ & $\begin{array}{l}\mathrm{Hb} \\
\mathrm{g} / \mathrm{dL}\end{array}$ & \multicolumn{2}{|c|}{$\underset{\%}{\mathrm{Ht}}$} & $\begin{array}{c}\text { WBC } \\
/ \mu \mathrm{L} \\
\end{array}$ & \multicolumn{2}{|c|}{$\begin{array}{cc}\text { Plt } & \text { A } \\
\times 10^{4} / \mu \mathrm{L} & \mathrm{I} \\
\end{array}$} & $\begin{array}{l}\text { AST } \\
\text { IU/L }\end{array}$ & $\begin{array}{c}\text { ALT } \\
\text { IU/L }\end{array}$ & $\begin{array}{c}\mathrm{LDH} \\
\mathrm{IU} / \mathrm{L}\end{array}$ & $\begin{array}{l}\text { T.Bil } \\
\mathrm{mg} / \mathrm{dL}\end{array}$ & $\begin{array}{c}\text { Glucose } \\
\text { mg/dL }\end{array}$ & e $\begin{array}{c}\mathrm{CRP} \\
\mathrm{mg} / \mathrm{dL}\end{array}$ \\
\hline Case 1 & 447 & 13.1 & 42.2 & & 5300 & 28. & & 19 & 10 & 187 & 0.6 & 86 & 0.19 \\
\hline Case 2 & 470 & 15.3 & 46.5 & & 6200 & 32. & & 23 & 20 & 192 & 0.6 & 92 & 0.03 \\
\hline Case 3 & 407 & 12.5 & 39.4 & & 5400 & $16 .^{\prime}$ & & 33 & 12 & 226 & 0.9 & 107 & 0.20 \\
\hline Case 4 & 445 & 13.3 & 41.5 & & 6600 & 17. & & 34 & 22 & 242 & 0.5 & 91 & 2.23 \\
\hline \multirow[t]{2}{*}{ Case 5} & 402 & 12.7 & 37.4 & & 5600 & 21. & & 18 & 13 & 127 & 0.5 & 100 & 4,96 \\
\hline & $\begin{array}{l}\text { BUN } \\
\mathrm{mg} / \mathrm{dL}\end{array}$ & $\begin{array}{c}\mathrm{Cr} \\
\mathrm{mg} / \mathrm{dL}\end{array}$ & $\begin{array}{r}\mathrm{TP} \\
\mathrm{g} / \mathrm{dL}\end{array}$ & $\begin{array}{l}\text { Alb } \\
\text { g/dL }\end{array}$ & & $\begin{array}{l}\text { loblin } \\
\mathrm{dL}\end{array}$ & $\begin{array}{c}\mathrm{IgG} \\
\mathrm{mg} / \mathrm{dL}\end{array}$ & & $\begin{array}{l}\mathrm{IgA} \\
\mathrm{ng} / \mathrm{dL}\end{array}$ & $\begin{array}{l}\mathrm{IgM} \\
\mathrm{mg} / \mathrm{dL}\end{array}$ & $\begin{array}{l}\text { ANA } \\
\text { fold }\end{array}$ & $\begin{array}{c}\mathrm{RF} \\
\mathrm{IU} / \mathrm{mL}\end{array}$ & $\begin{array}{l}\mathrm{CP}-\mathrm{Ab} \\
\mathrm{U} / \mathrm{mL}\end{array}$ \\
\hline Case 1 & 10 & 0.62 & 7.2 & - & & - & - & & - & - & - & - & - \\
\hline Case 2 & 14 & 0.80 & 7.0 & 4.4 & & .13 & - & & - & - & - & - & - \\
\hline Case 3 & 15 & 0.70 & 7.6 & 4.1 & & .53 & 1489 & & $63.2 \quad$ & 263.2 & 40 & 1.0 & 1.1 \\
\hline Case 4 & 20 & 1.05 & 7.1 & 3.9 & & .71 & 1679 & & 71.5 & 114.3 & 40 & 7.0 & $<0.5$ \\
\hline Case 5 & 15 & 0.98 & 6.7 & 3.5 & & - & 1142 & & 21.6 & 49.1 & 40 & 3.0 & 0.5 \\
\hline
\end{tabular}

TP: total protein, ANA: anti-nuclear antibody, RF: rheumatoid factor, CCP-Ab: anti-

CCP antibody, -: not examined

materials for histological analysis were available, and herein report our findings.

This study was approved by the Himeji Medical Center Review Board (26-4). Among the NTM disease patients treated at Himeji Medical Center, Japan, whose diseases were diagnosed in accordance with the ATS/IDSA guidelines (1) over a 10-year period (January 2003-November 2013), we extracted the patients who had undergone a biopsy or surgical resection while being diagnosed or treated for their NTM diseases. Among them, patients with histological OP were considered to be candidates for the present study. Finally, we excluded patients with diseases wellknown to cause OP. The clinical and radiological features of the subjects were examined retrospectively based on their medical records.

\section{Case Reports}

Out of 560 NTM disease patients, 98 patients $(55$ men and 43 women) underwent histological examinations (biopsy, 42; surgical resection, 65). Their median age was 65 (range 21-84) years, and their main shadows consisted of nodular shadows (49 cases), infiltrating shadows (17 cases), fibrocavitary shadows (15 cases) and others. Out of the 98 patients, 11 cases showed OP histologically, but six of them had diseases that are well-known to cause OP [idiopathic interstitial pneumonia (three patients), lung cancer (two patients) and chronic rheumatoid arthritis (one patient)]. The remaining five patients were included as the subjects of the present study. Based on their chest images, we classified these patients into the nodule group (three patients) and the infiltrate group (two patients). The patients' laboratory data are shown in the Table.

Fig. 1 shows representative CT images and histological features of the patients in the nodule group. Mycobacterium avium was detected in a culture from the resected specimen (Case 1) or bronchial washing (Cases 2 and 3). The nodules of Cases 1 and 2 were resected because of a suspicion of lung cancer, and Case 3 was successfully treated with rifam- picin, clarithromycin and ethambutol (RECAM).

Cases 4 and 5 belonged to the infiltrate group. Case 4 (Fig. 2) was a 73-year-old man. His chest X-ray that had been taken during a routine health check-up eighteen months earlier showed no abnormalities. At presentation, he complained of fever, and infiltrates were seen. The bronchoalveolar lavage fluid (BALF) obtained at the right upper lobe demonstrated increased lymphocytes (50\%), with a CD4/CD8 ratio of 5.86, and was culture-positive for Mycobacterium avium. The transbronchial lung biopsy (TBLB) specimen showed epithelioid cell granuloma and OP (Fig. 3A, B). Because the shadows worsened, prednisolone was introduced, and the shadows began to improve within one week. Thereafter, RECAM was added, but a cavity remained. As the cavity did not improve, right upper lobectomy was performed. The resected material revealed acidfast bacilli-positive epithelioid cell granulomas with necrosis, and there was fibrosing granulation in the peripheral region (Fig. 3C). He was treated with RECAM for eight months without relapse.

Case 5 (Fig. 4) was a 66-year-old woman who complained of cough, and her CT showed multiple cavitary nodules. Bronchial washing revealed Mycobacterium abscessus on culture. The shadows improved following treatment with imipenem/cilastatin, amikacin, rifampicin and clarithromycin, and she was subsequently treated with RECAM. She suddenly developed a cough and fever with infiltrates, accompanied by an air bronchogram in the right upper lobe. BALF obtained at the right upper lobe revealed increased lymphocytes (79\%), with a CD4/CD8 ratio of 1.36 , and yielded no significant bacteria on culture. TBLB showed OP, but not epithelioid cell granuloma (Fig. 5).

The shadows in this patient migrated. BALF collected from the left upper lobe was positive for Mycobacterium $a b$ scessus, but no other organism was detected. Prednisolone was administered, and a dramatic improvement of the shadows was seen. However, with tapering of prednisolone, the shadows relapsed. With an increased dose of prednisolone and gradual tapering, the shadows became stable with $5 \mathrm{mg}$ 


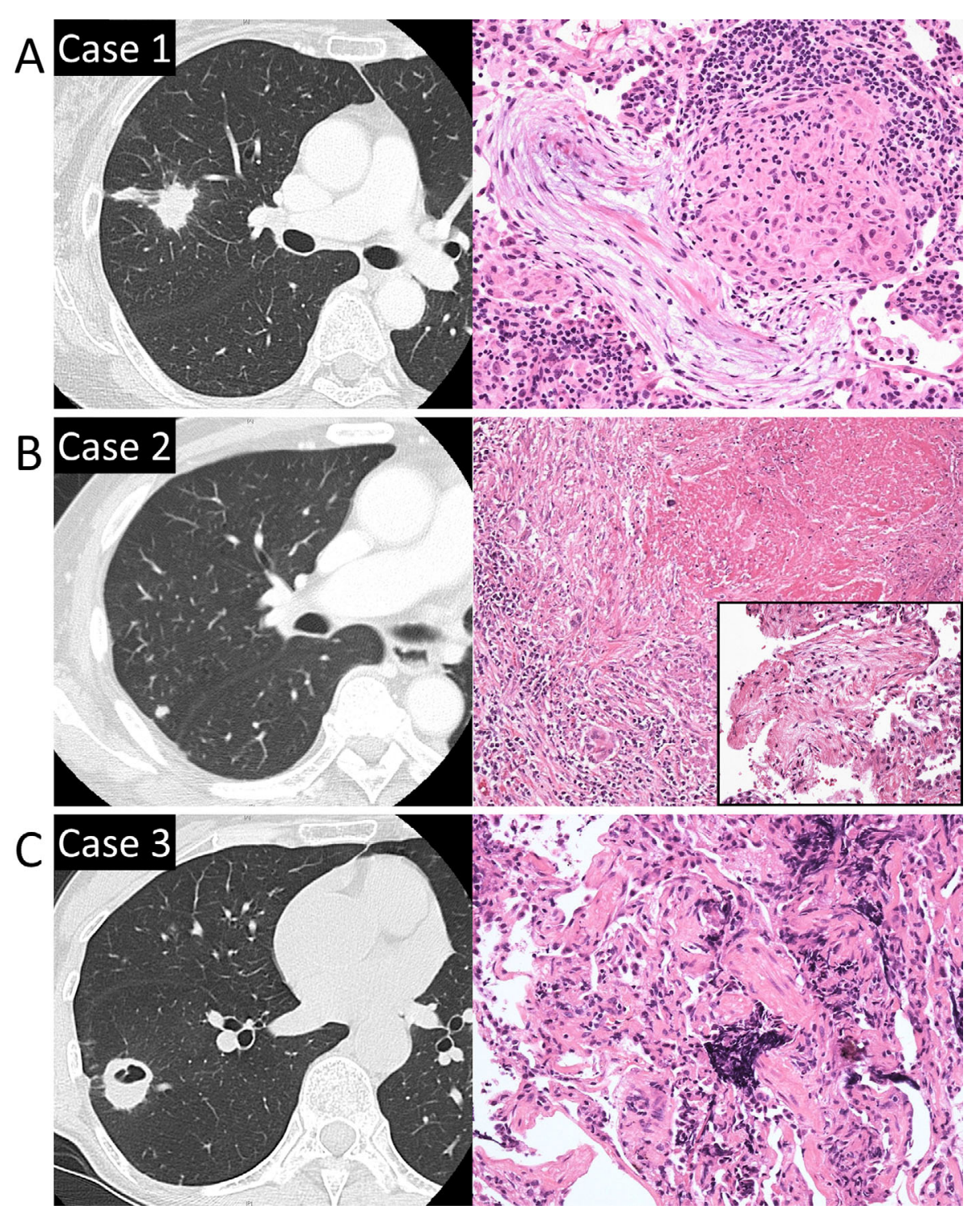

Figure 1. Representative CT images and histological findings. A: Case 1. A 66-year-old woman complained of cough, and a nodular shadow was pointed out at the right S2-S3 (left). The resected material revealed organizing pneumonia with non-necrotizing epithelioid cell granuloma [right, Hematoxylin and Eosin (H\&E) staining, original $\times 100$ ]. B: Case 2. A CT from a 74-year-old man with a history of surgery for stage IA lung adenocarcinoma in the left upper lobe four years earlier. A small nodule at the right $\mathrm{S} 2$ was pointed out (left). The resected material showed epithelioid cell granulomas with necrosis, and organizing pneumonia around the granulomas (right, $\mathrm{H} \& \mathrm{E}$ staining, original $\times \mathbf{6 0}$; magnification of the inset, $\times 60$ ). C: Case 3. A 65-year-old woman complained of fever. CT showed a nodule with a cavity at the right S6 (left). Bronchial washing revealed Mycobacterium avium on culture, and a transbronchial lung biopsy showed organizing pneumonia but no granuloma (right, $H \& E$ staining, original $\times 100)$.

of prednisolone and RECAM.

\section{Discussion}

OP is a non-specific response to various stimuli or injuries that can be cryptogenic or associated with several causes, such as respiratory infections, connective tissue disease, drugs, radiation therapy, viral infections, myelodysplastic syndrome, aspiration pneumonia, organ transplantation, lung cancer and some occupations or environ- ments $(3,8,9)$.

Histologically, OP was seen in 11 out of our 98 NTM disease patients in whom histological materials were available. However, the coexistence of NTM and OP itself does not necessarily mean that nontuberculous mycobacteria (NTM) induce OP. For example, OP is often associated with rheumatoid arthritis (3), and NTM infections are relatively common in patients with rheumatoid arthritis (10); as a result, OP seems to be related to NTM disease. To strictly study the possibility of a direct relationship between NTM 
Case 4: 73-year-old male

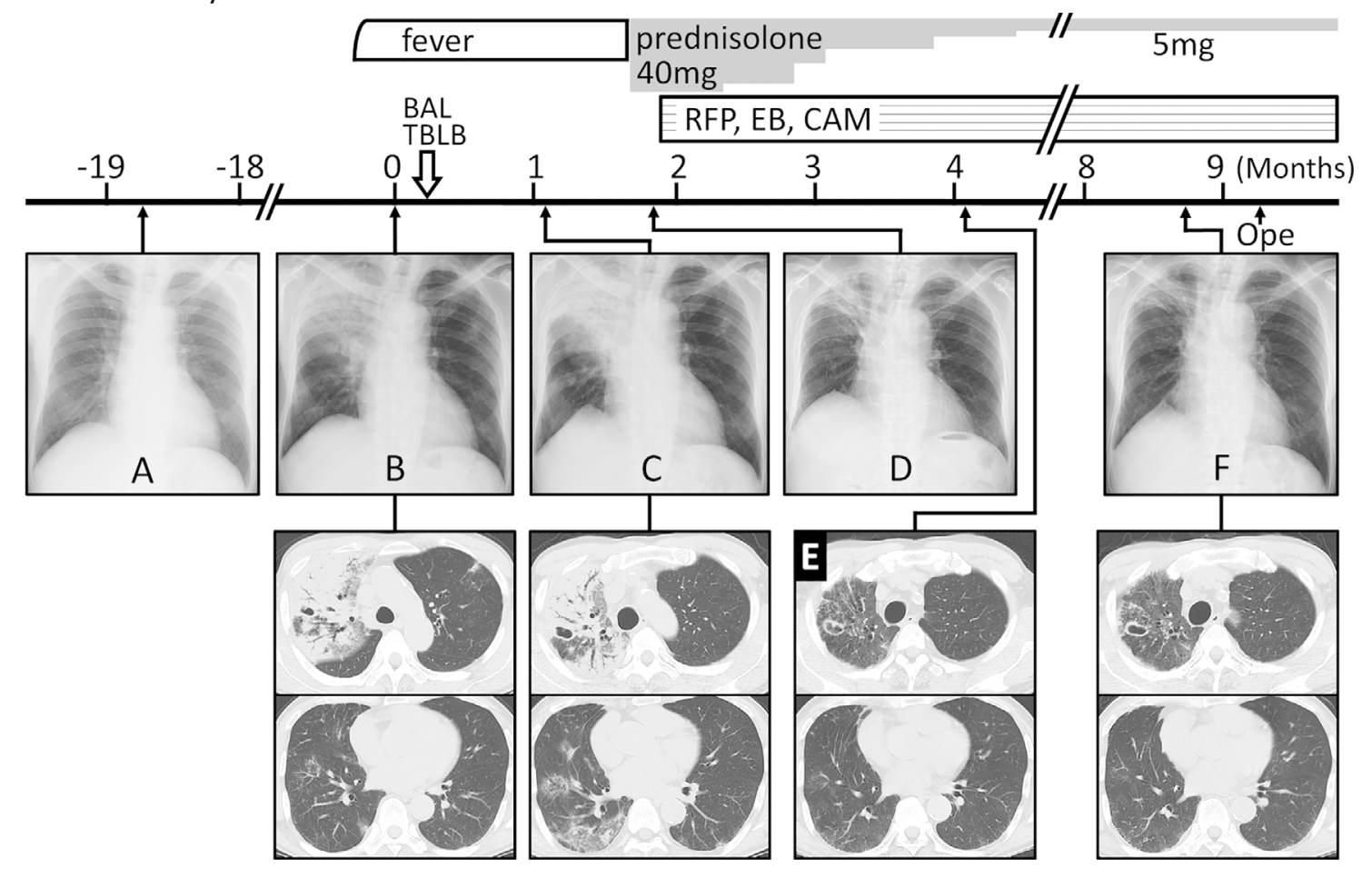

Figure 2. The clinical course of Case 4. A: A health check-up performed eighteen months before the development of symptoms, revealed no abnormalities on the chest $X$-ray. B: The patient complained of fever, and his chest X-ray and CT scan showed infiltrates with an air bronchogram in the right upper lobe, and ground glass opacities in both lung fields. The BALF was culture-positive for Mycobacterium avium. C: After being followed-up for one month, the shadows increased. D: After the introduction of a corticosteroid, the fever and shadows began to diminish within one week. E: After two months of therapy with prednisolone and anti-mycobacterial medication, almost all of the shadows had disappeared, leaving a cavity. F: An additional five months of the therapy could not improve the cavity; therefore, right upper lobectomy was performed. RFP: rifampicin, EB: ethambutol, CAM: clarithromycin, BAL: bronchoalveolar lavage, TBLB: transbronchial lung biopsy, Ope: surgical resection

and $\mathrm{OP}$, we excluded patients with possible OP-related diseases from our subjects, and five patients remained. Strictly speaking, in Case 5, we could not rule out the possibility that rifampicin or clarithromycin, which were administered throughout the treatment course, had induced the OP. However, as the OP was clinically controlled with only $5 \mathrm{mg}$ of prednisolone under the administration of RECAM, we believe that the drugs were not related to the development of $\mathrm{OP}$ in this case.

Our five subjects mainly had nodules or infiltrates, which was different from the common constitution of NTM diseases, which are mainly fibrocavitary and nodular/bronchiectatic. This does not necessarily mean that OP more readily accompanies specific types of NTM diseases, because our subjects were extracted from the subpopulation of NTM disease patients who had an indication for biopsy or surgical resection, which might have biased the type of disease.

Our infiltrate group patients (Cases 4 and 5) showed common clinical features. They both showed acute symptoms of fever or cough with infiltrating shadows accompanied by air bronchogram, which improved rapidly by treatment with a corticosteroid and anti-mycobacterial therapy. Similar cases have been reported in the literature. For example, Hamada et al. (4) reported the case of a 67-year-old woman with fever and dyspnea. Her sputa culture confirmed Mycobacterium intracellulare, and TBLB revealed OP that rapidly improved clinically with corticosteroid administration. The chest CT findings of Hamada's case resembled those of our infiltrate group patients.

Jones et al. (5) reported the case of a 58-year-old woman with Mycobacterium avium complex infection, and Starobin et al. (6) described an 85-year-old woman with Mycobacterium kansasii infection with biopsy-proven OP, both of which were successfully treated with anti-mycobacterial medication and steroids. In these reports, the authors speculated that OP was induced secondary to NTM disease or that OP was one of the clinicopathologic features of NTM diseases. Two other reports described a 79-year-old man and 50-year-old woman with similar features, except that their OP was diagnosed only clinically, but not histologically $(11,12)$. Waller et al. (13) reported the case of a 32year-old woman with Mycobacterium avium complex dis- 

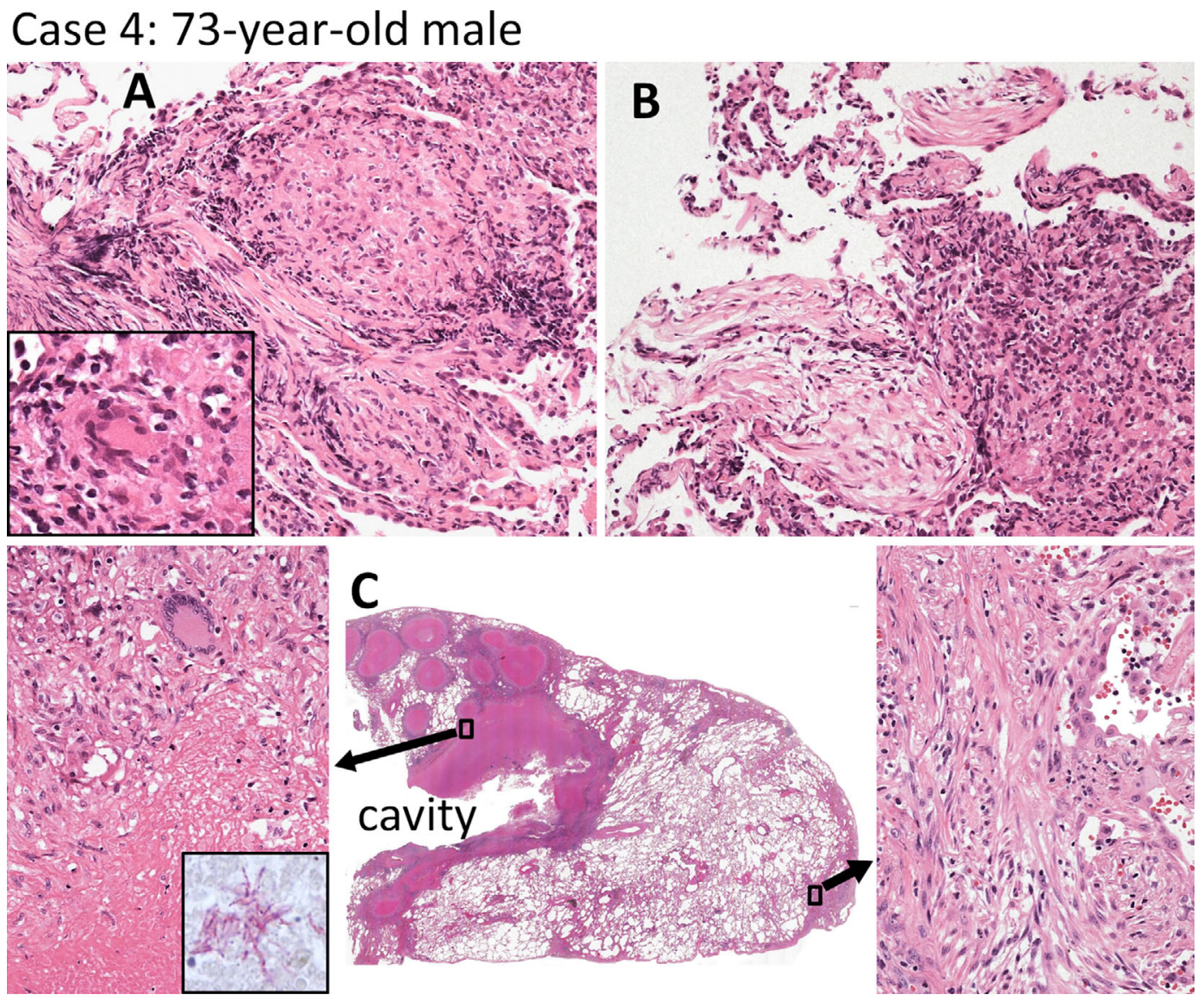

Figure 3. Case 4. A, B: A transbronchial lung biopsy specimen revealed epithelioid cell granulomas with giant cells $(A, H \& E$ staining, original $\times 100$ and $\times 200)$ and organizing pneumonia $(B, H \& E$ staining, original $\times 100)$. C: The resected material revealed a cavity. Acid-fast bacilli-positive epithelioid cell granulomas with necrosis were seen in the cavitary wall, and fibrosing granulation was seen in the peripheral region (left: $H \& E$ staining, original $\times 100$, inset: Ziehl-Neelsen, original $\times 1,000$, middle: H\&E staining, original $\times 1$, right: H\&E staining, original $\times 100)$.

ease with similar shadows and a similar clinical course, although the authors did not mention OP. The close resemblance of these cases warrants us to consider the possibility that they represent a common disease entity.

On the other hand, because we could not recognize a difference in the pathological features between the nodule group and infiltrate group, we suppose that the patients with infiltrates did not have an independent disease, but that the accompanying OP may have modified the clinical features of the NTM diseases, and that cases with severe OP reactions may have acute symptoms and vast infiltrates that require corticosteroids.

Verma et al. (14) reported two cases of hot tub lung (HTL), but one of them had bilateral lower lobe consolidation with air bronchogram, just like our infiltrate group patients. Although they diagnosed their patient with HTL, they discussed the possibility of the diagnosis of OP. The typical histological feature of HTL is bronchiolocentric nonnecrotizing granulomatous inflammation, but some cases have been reported to have OP histologically (15).

Given these previous studies, the relationship between our infiltrate group and HTL should be discussed. The typical radiological findings of HTL are essentially diffuse infiltrates with prominent nodularity (1), which are largely different from the infiltrating shadows with air bronchogram seen in our infiltrate group. Our Case 4 and the other reported cases $(5,13)$ showed cavitary NTM lesions, and in our Case 5 and Starobin's case (6), the detection of NTM preceded the appearance of infiltration shadows by one to two years. These findings also are inconsistent with HTL.

The CD4/CD8 ratio in the BALF of HTL patients is generally high (16), whereas that of cryptogenic OP patients tends to be low $(17,18)$. The elevated CD4/CD8 ratio in Case 4 may suggest the possibility of HTL. However, as the CD4/CD8 ratio in the BALF in patients with NTM disease has been reported to be normal or elevated $(19,20)$, the CD4/CD8 ratio alone cannot differentiate between OP with NTM and HTL.

In conclusion, NTM diseases are sometimes accompanied by OP histologically, and some such cases have clinical features that are characterized by acute or subacute symptoms, infiltrating shadows with air bronchogram and dramatic improvement following treatment with a corticosteroid and anti-NTM therapy. These striking clinical features and the 
Case 5: 66-year-old female

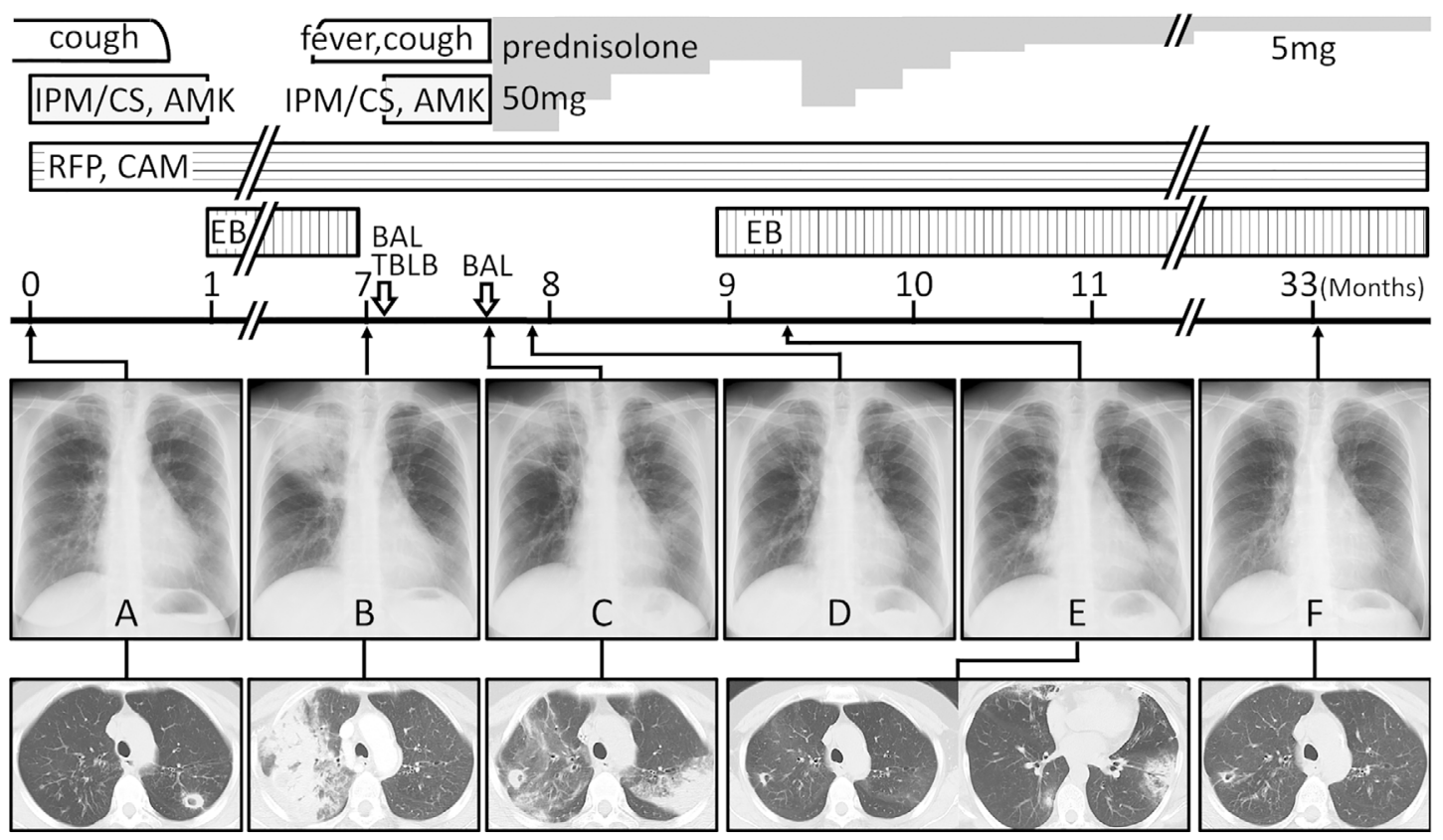

Figure 4. The clinical course of Case 5. A: A CT scan showed multiple cavitary nodules, the BALF was positive for Mycobacterium abscessus on culture and the patient was treated with anti-mycobacterial therapy. B: Infiltrates accompanied by an air bronchogram appeared suddenly in the right upper lobe. C: IPM/CS with AMK was introduced, and the infiltrates in the right upper lobe diminished, leaving a cavitary nodule, but a new infiltrate appeared in the left upper lobe. D: The infiltrates rapidly improved by corticosteroid administration. E: With the tapering of prednisolone, the shadows relapsed; they were successfully treated with an increased dose of prednisolone. $F$ : The dose of prednisolone could be reduced to $5 \mathrm{mg}$ daily without relapse by gradual tapering. IPM/CS: imipenem/ cilastatin, AMK: amikacin, RFP: rifampicin, CAM: clarithromycin, EB: ethambutol, BAL: bronchoalveolar lavage, TBLB: transbronchial lung biopsy

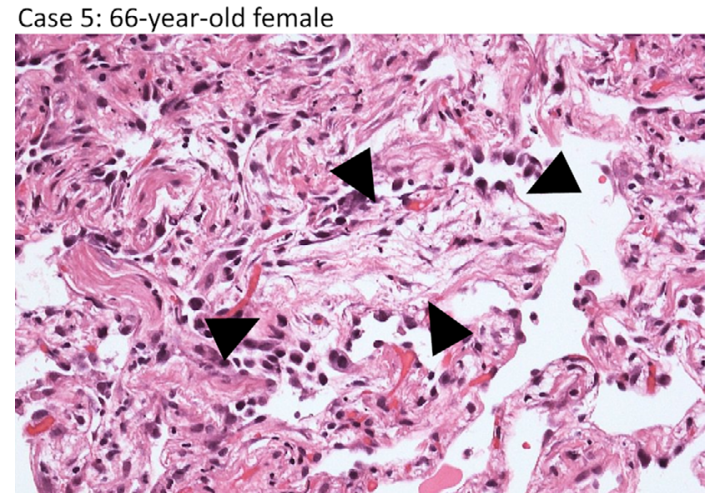

Figure 5. Case 5. A transbronchial lung biopsy specimen showed organizing pneumonia (arrow head, H\&E staining, original $\times \mathbf{1 0 0}$ ).

response to corticosteroid treatment are not currently described in the ATS/IDSA statement on nontuberculous mycobacterial diseases, and clinicians should be aware of such cases when deciding on the course of treatment.

The authors state that they have no Conflict of Interest (COI).

\section{References}

1. Griffith DE, Aksamit T, Brown-Elliott BA, et al. An official ATS/ IDSA statement: diagnosis, treatment, and prevention of nontuberculous mycobacterial diseases. Am J Respir Crit Care Med 175: 367-416, 2007.

2. Epler GR. Bronchiolitis obliterans organizing pneumonia: definition and clinical features. Chest 102 (Suppl): 2S-6S, 1992.

3. Epler GR. Bronchiolitis obliterans organizing pneumonia, 25 years: a variety of causes, but what are the treatment options? Expert Rev Respir Med 5: 353-361, 2011.

4. Hamada K, Nagai S, Hara Y, Hirai T, Mishima M. Pulmonary infection of Mycobacterium avium-intracellulare complex with simultaneous organizing pneumonia. Intern Med 45: 15-20, 2006.

5. Jones RM, Dawson A, Evans EN, Harrison NK. Co-existence of organising pneumonia in a patient with Mycobacterium avium intracellulare pulmonary infection. Monaldi Arch Chest Dis 71: 7680, 2009.

6. Starobin D, Guller V, Gurevich A, Fink G, Huszar M, Tal S. Organizing pneumonia and non-necrotizing granulomata on transbronchial biopsy: coexistence or bronchiolitis obliterans organizing pneumonia secondary to Mycobacterium kansasii disease. Respir Care 56: 1959-1961, 2011.

7. Marchevsky A, Damsker B, Gribetz A, Tepper S, Geller SA. The spectrum of pathology of nontuberculous mycobacterial infections in open-lung biopsy specimens. Am J Clin Pathol 78: 695-700, 
1982.

8. Romero S, Barroso E, Rodriguez-Paniagua M, Aranda FI. Organizing pneumonia adjacent to lung cancer: frequency and clinicopathologic features. Lung Cancer 35: 195-201, 2002.

9. Yousem SA, Faber C. Histopathology of aspiration pneumonia not associated with food or other particulate matter: a clinicopathologic study of 10 cases diagnosed on biopsy. Am J Surg Pathol 35: 426-431, 2011.

10. Brode SK, Jamieson FB, Ng R, et al. Risk of mycobacterial infections associated with rheumatoid arthritis in Ontario, Canada. Chest 146: 563-572, 2014.

11. Khanlari B, Burri E, Mihatsch F, Flückiger U, Battegay M. Mycobacterium avium pneumonia in an HIV negative patient. Internist (Berl) 48: 527-531, 2007.

12. Okazaki A, Takato H, Fujimura M, Ohkura N, Katayama N, Kasahara K. Successful treatment with chemotherapy and corticosteroids of pulmonary Mycobacterium abscessus infection accompanied by pleural effusion. J Infect Chemother 19: 964-968, 2013.

13. Waller EA, Roy A, Brumble L, Khoor A, Johnson MM, Garland JL. The expanding spectrum of Mycobacterium avium complexassociated pulmonary disease. Chest 130: 1234-1241, 2006.

14. Verma G, Jamieson F, Chedore $P$, et al. Hot tub lung mimicking classic acute and chronic hypersensitivity pneumonitis: two case reports. Can Respir J 14: 354-356, 2007.

15. Khoor A, Leslie KO, Tazelaar HD, Helmers RA, Colby TV. Diffuse pulmonary disease caused by nontuberculous mycobacteria in immunocompetent people (hot tub lung). Am J Clin Pathol 115: 755-762, 2001.

16. Marras TK, Wallace RJ Jr, Koth LL, Stulbarg MS, Cowl CT, Daley CL. Hypersensitivity pneumonitis reaction to Mycobacterium avium in household water. Chest 127: 664-671, 2005.

17. Izumi T, Kitaichi M, Nishimura K, Nagai S. Bronchiolitis obliterans organizing pneumonia. Clinical features and differential diagnosis. Chest 102: 715-719, 1992.

18. Poletti V, Cazzato S, Minicuci N, Zompatori M, Burzi M, Schiattone ML. The diagnostic value of bronchoalveolar lavage and transbronchial lung biopsy in cryptogenic organizing pneumonia. Eur Respir J 9: 2513-2516, 1996.

19. Yamazaki Y, Kubo K, Sekiguchi M, Honda T. Analysis of BAL fluid in $M$. avium-intracellulare infection in individuals without predisposing lung disease. Eur Respir J 11: 1227-1231, 1998.

20. Heurlin N, Bergström SE, Andersson J, Christensson M, Christensson B. Lack of T-lymphocytosis and poor interferon gamma production in BAL fluid from HIV-negative immunocompetent patients with pulmonary non-tuberculous mycobacteriosis. Scand J Infect Dis 30: 339-343, 1998.

(C) 2015 The Japanese Society of Internal Medicine http://www.naika.or.jp/imonline/index.html 